\title{
Lidil
}

Revue de linguistique et de didactique des langues

$63 \mid 2021$

Littératie numérique et didactique des langues et des cultures

\section{Littératie numérique et expertise distribuée : inscription et révision lors d'une écriture collaborative en présentiel}

Digital Literacy and Distributed Expertise: Transcription and Revision in Face-to-face Collaborative Writing

Marie-Odile Hidden et Henri Portine

\section{(2) OpenEdition}

Journals

Édition électronique

URL : http://journals.openedition.org/lidil/8913

DOI : 10.4000/lidil.8913

ISSN : 1960-6052

Éditeur

UGA Éditions/Université Grenoble Alpes

Édition imprimée

ISBN : 978-2-37747-283-3

ISSN : $1146-6480$

Référence électronique

Marie-Odile Hidden et Henri Portine, «Littératie numérique et expertise distribuée : inscription et révision lors d'une écriture collaborative en présentiel », Lidil [En ligne], 63 | 2021, mis en ligne le 30 avril 2021, consulté le 30 avril 2021. URL : http://journals.openedition.org/lidil/8913 ; DOI : https:// doi.org/10.4000/lidil.8913

Ce document a été généré automatiquement le 30 avril 2021.

(c) Lidil 


\title{
Littératie numérique et expertise distribuée : inscription et révision lors d'une écriture collaborative en présentiel
}

\author{
Digital Literacy and Distributed Expertise: Transcription and Revision in \\ Face-to-face Collaborative Writing
}

Marie-Odile Hidden et Henri Portine

\section{Introduction}

1 Depuis quelques années, nous tentons de changer notre façon d'appréhender l'apprentissage de l'écrit en langue étrangère (en l'occurrence en français) en passant (comme d'autres) d'une conception articulant compréhension et productions écrites à une conception qui considère lecture et écriture comme imbriquées et comme mode d'expression d'une culture de l'écrit historiquement et géographiquement déterminée, ce que nous nommerons "la littératie (définition ci-dessous) avec une composante numérique (parce que le papier reste l'une des matérialisations de l'écrit) ». Dès lors, notre problématique a évolué vers la question suivante : l'adoption d'une approche de type "littératie numérique " qui suppose un réseau (ou un ensemble de réseaux) favorise-t-elle la production d'écrit, c'est-à-dire l'acquisition d'une compétence à se construire une posture de scripteur au sein d'une communauté donnée et en fonction d'un objectif explicité? Nous avons choisi la modalité collaborative parce qu'elle modélise (ou simule, mais au sens technique) l'activité mentale (les questionnements, voire les débats internes) de tout sujet aux prises avec la lecture ou l'écriture d'un texte complexe: tenir compte d'opinions divergentes, s'acclimater à un auditoire et à un contexte précis, choisir en écriture une formulation et en lecture une interprétation adéquates, etc. Pour répondre à la question ci-dessus, nous avons constitué un dispositif numérique reposant sur un 
double réseau en présentiel : celui du débat oral en vue d'une production écrite, celui de la production effective du texte écrit à plusieurs.

2 Cette problématique, assez générale, se décline en sous-problématiques dont l'une formera l'objet de cet article ${ }^{1}$ (d'autres ayant déjà donné lieu ou devant donner lieu à d'autres publications $\left.{ }^{2}\right)$ : notre dispositif didactique favorise-t-il la constitution par chaque apprenant de sa posture de sujet-scripteur? Quels en sont les succès et les échecs? Pour évaluer ce positionnement de sujet-scripteur, nous recourons à deux paramètres: l'inscription (réalisation de la trace écrite) et les (hétéro-)révisions. Précisons ce que nous entendons par littératie (numérique) et analysons notre corpus en fonction de ces deux paramètres.

\section{Literacy et littératie numérique}

3 Nous avons dit en introduction que notre toile de fond depuis quelques années était engendrée par le concept de littératie. Pour bien saisir cette évolution (qui est en fait un changement de paradigme au sens de Kuhn, 19703), parce que l'emploi des mots véhicule toujours des modes de pensée, il nous faut nous livrer à de rapides considérations épistémologiques.

4 Le concept de littératie est d'origine anglo-saxonne (literacy). Au départ (1880, Oxford English Dictionary), le terme anglais désigne alors ce qu'on appelle en français, à peu près à la même époque, alphabétisation (pour deux types de considérations: développement de l'industrialisation et homogénéisation de la nation). À la fin du $\mathrm{xx}^{\mathrm{e}}$ siècle, face à la prise de conscience de l'illettrisme aux États-Unis, au Royaume-Uni et en Australie ${ }^{4}$ (mais aussi, de façon un peu différente, en France), l'anglais literacy va signifier « s'approprier la culture de l'écrit » et un terme français littératie va s'imposer (mais sans être, pour l'instant, adopté par l'Académie française). Or quelle est la différence fondamentale entre literacy et alphabétisation? Le premier concept a une visée englobante, la culture de l'écrit, alors que le second désigne deux phases, apprendre à lire et apprendre à écrire. C'est sans doute pour cette raison qu'il n'a jamais été construit en français de termes positifs opposé à illettrisme (ce que sont literacy et literate), lettré n'étant pas l'opposé d'illettré en français.

Donnons une définition de littératie, inspirée de celle de l'OCDE (2000, p. x). La littératie est l'aptitude à comprendre et à produire de l'information écrite (au sens général des psycholinguistes : données, points de vue, etc.) dans la vie courante et professionnelle, en vue, pour le lecteur-scripteur, d'être un élément actif d'une communauté donnée (qui peut être de divers ordres, quelqu'un qui travaille à l'international devant s'accommoder aux deux cultures de l'écrit en présence: celle de sa communauté et celle de la communauté étrangère).

Concept englobant et plastique, la literacy/littératie (nous conservons les deux termes parce que nous traitons ici du concept d'origine anglo-saxonne mais en cours d'adoption depuis les années 1990 en contexte francophone) est aujourd'hui marquée ou non (selon les auteurs et leurs objectifs) par le numérique, c'est-à-dire l'alliance des algorithmes (issus des années 1930), du développement des réseaux électroniques (années 1980) et du langage XML (profilé à partir de 1975 mais véritablement constitué vers 1995). À partir de la littérature plus ou moins technique, nous avons été amenés à 
considérer que la literacy/littératie (numérique ou non) pouvait s'envisager à partir de quatre points de vue (ce qui nous permettra de situer notre recherche) :

a. une dimension historique, sociologique et anthropologique, la culture de l'écrit étant liée à sa contextualisation (sociale, géographique, culturelle; cf. Goody \& Watt, $1963^{5}$; Privat \& Kara, 2006), d'où le problème du rapport entre culture de l'oral et culture de l'écrit (Masseron \& Privat, 2019) ;

b. la constitution d'un «fonds culturel » qui a correspondu au rêve romantique de l'avènement de l'Internet (le grand partage du savoir) ; Lanham (1995) et Gilster (1997, 2006), qui étaient des essayistes et n'étaient spécialisés ni dans le numérique ni dans la literacy, ont considéré qu'un «nouveau monde » (digital world pour le premier, electronic city pour le second) était né, plus proche de la culture de l'oralité que de la culture de l'écrit-papier, celui de «l'information virtuelle»;

c. l'étude de la diffusion de l'information et des savoirs en sciences de la documentation (en anglais, Library and Information Science); Bawden (2001) a fait une très minutieuse étude suivie d'autres publications (Bawden \& Robinson, 2002 ; Bawden, 2008);

d. l'évolution des dispositifs éducatifs de maitrise de l'écrit en contexte devenu numérique ; il fallait repenser les habiletés à l'œuvre (Lankshear \& Knobel, 2003, 2006; Martin \& Madigan, 2006) ${ }^{6}$ et les dispositifs (Martin \& Grudziecki, 2006, dans le cadre d'un projet européen pour la mobilité); ce qui ressort de ces travaux, c'est l'importance de l'expertise distribuée au sein de réseaux, le fait que le concept de littératie implique d'inscrire les activités dans la textualisation, la nécessaire construction de relations de collaboration et la coréalisation des tâches, la nécessité de se situer par rapport à une communauté donnée ${ }^{7}$; la nécessité d'un nouveau rôle, celui de tuteur ${ }^{8}$, est aussi apparue (Depover et coll., 2011).

7 La didactique des langues (maternelle et étrangères ou secondes) hérite plus ou moins de tous ces points de vue, et surtout du point (d). En langue étrangère, la problématique de la littératie numérique est déjà vivante (entre autres: Warschauer, 1999; Kern, 2000 ; Mangenot, 2000 ; Thorne, 2008; Grosbois, 2012 ; Ollivier, 2018). Certains de ces auteurs n'utilisent pas le terme literacy/littératie, mais recourent à des notions propres à cette problématique (rapport lire/écrire, réseaux sociaux). Nous nous situons donc dans ce contexte.

8 Nous avons donc choisi un outil permettant de travailler en réseau: Framapad ${ }^{\circ}$ Les apprenants regroupés par trois débattent oralement et écrivent en collaboration, d'où des propositions de verbalisation, des préformulations à l'oral ainsi que des auto/ hétéro-dictées, ce qui crée à proprement parler des "situations oralographiques", comme le notent à juste titre Gaulmyn et coll. (2001, p. 10). Comme nous allons le voir et contrairement à la description que fait Gaulmyn (2005) de séances d'écriture à deux sur papier, ces manifestations orales, dans notre dispositif avec Framapad ${ }^{\circ}$, ne sont pas le plus souvent préalables à l'écriture collaborative mais concomitantes ou quasiconcomitantes (ce n'est pas de la préverbalisation ${ }^{9}$ ) ; l'apprenant se responsabilise soit de façon proactive soit en réagissant.

Nous allons maintenant présenter dispositif et corpus, puis nos analyses qui montrent comment les inscriptions faisant texte et les révisions mutuelles s'articulent ou non aux négociations entre pairs dans l'écriture collaborative à l'aide d'outils numériques. 


\section{Dispositif et corpus}

\subsection{Dispositif et objectifs de recherche}

10 Notre dispositif d'écriture collaborative en présentiel auquel a été ajoutée une médiation ${ }^{10}$ numérique (Framapad ${ }^{\circ}$ ) a été mis en place au sein d'un semestre (12 semaines) de cours de français langue étrangère visant à améliorer les compétences écrites en compréhension et en production (dans une optique de littératie, cf. ci-avant) de 15 apprenants adultes provenant de 10 pays différents, de niveau B1-B2. Il obéit aux objectifs suivants :

- observer comment les apprenants s'approprient des outils d'écriture numériques en présentiel (Framapad ${ }^{\circ}$, mais aussi Word ${ }^{\circ}$ ) ;

- observer comment les apprenants réalisent le processus de collaboration ;

- observer les négociations entre co-apprenants lors d'une tâche de rédaction collaborative.

Voici les paramètres de notre dispositif qui en découlent :

- dimension spatiale : le cours a lieu dans une salle où tous les sièges, mobiles et pourvus de rabats pour poser documents et ordinateur portable, sont regroupés par trois ;

- dimension communicationnelle : interactions entre les apprenants regroupés par trois (ces interactions sont enregistrées et font partie du corpus) ; les consignes sont données au début par l'enseignant qui passe ensuite entre les groupes (ce qui peut entrainer des questions) ;

- dimension pédagogique et temporelle : tout au long du semestre, tâches de compréhension écrite et tâches de production écrite alternent et sont étroitement corrélées ; les consignes d'écriture visent en effet à faire réagir les apprenants à la lecture d'un article de presse (texte 1 et texte $3^{11}$ ) ou à un message posté sur un forum (texte 2) ;

- dimension technologique: nous avons choisi le logiciel Framapad $^{\circ}$ pour l'écriture collaborative en le faisant utiliser en présentiel afin de pouvoir observer les interactions entre les étudiants pendant la rédaction ainsi que les enregistrer en vue d'une analyse détaillée.

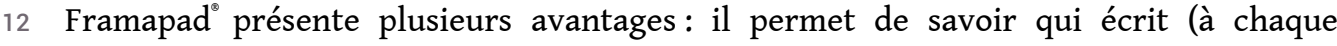
scripteur est assignée une couleur d'écriture) ; il comporte un historique dynamique original qui permet de visionner en accéléré l'élaboration du texte dans ses moindres détails. Les étudiants ont utilisé cet outil pour rédiger trois textes ${ }^{12}$, en début, au milieu et en fin de semestre. Afin de pouvoir mieux saisir les spécificités de Framapad ${ }^{\circledR}$ par contraste, une tâche d'écriture sous Word ${ }^{\circledR}$ a également été donnée aux apprenants.

13 Nous travaillons sur la compréhension et la production d'argumentations: les apprenants sont appelés à donner leur opinion sur un article de presse ou/et sur des sujets polémiques, ce qui nécessite la gestion d'éventuelles divergences; il y a ainsi des allers et retours entre lecture et écriture ${ }^{13}$.

\subsection{Corpus d'observation et méthodologie d'analyse}

Constitué de fichiers numériques et d'enregistrements audios, le corpus bimodal comprend :

- les 15 textes rédigés en collaboration avec Framapad et exportés au format PDF ;

- les 15 textes rédigés en collaboration avec Framapad annotés par l'enseignant;

- les historiques dynamiques de l'écriture des textes ; 
- les 5 textes rédigés en collaboration sous Word ${ }^{\circ}$;

- les 5 textes rédigés en collaboration sous Word ${ }^{\circledR}$ annotés par l'enseignant ;

- les enregistrements audios des interactions entre apprenants lors de l'écriture collaborative, soit près de 11 heures d'enregistrement ;

- les textes comprenant les commentaires métacognitifs des apprenants ;

- deux questionnaires remplis par les étudiants : un questionnaire de début de cours sur leurs pratiques de lecture et d'écriture en langue maternelle et en langue(s) étrangère(s) ; un questionnaire de fin de cours sur leurs pratiques de lecture et d'écriture en français ;

- des observations réalisées in vivo.

15 Au point de vue méthodologique, nous avons réalisé un descriptif détaillé de l'évolution de chaque texte à partir de son historique dynamique et des enregistrements audios (déroulement du processus d'écriture : qui écrit quoi ; qui prend quelle initiative ; qui propose quoi, etc.). Les textes annotés par l'enseignant nous ont servi à évaluer la qualité des textes produits en collaboration.

Les consignes données étaient très peu contraignantes : une très grande liberté était laissée aux apprenants pour constituer les groupes ${ }^{14}$ et pour organiser et réaliser le travail en collaboration. Les apprenants ont profité de cette liberté d'action pour se saisir des fonctionnalités de Framapad ${ }^{\circ}$ et réellement collaborer entre eux.

\section{Collaboration avec Framapad $^{\circledR}$ : inscription et révisions}

17 Gaulmyn (2005, p. 25) a décrit la «rédaction conversationnelle » (c'est-à-dire les échanges liés aux phases de production lors de l'écriture d'un texte à plusieurs) sur papier, en distinguant des phases: élaboration pré-rédactionnelle, élaboration rédactionnelle, inscription $d u$ fragment de texte adopté, contrôle et révision. Nous avons retenu et développé la notion d'inscription. La littératie numérique se caractérisant, comme nous l'avons vu, par l'expertise distribuée au sein des réseaux et la coréalisation des tâches, nous avons cherché à évaluer le degré de collaboration entre les co-apprenants aux différents niveaux suivants : recherche d'idées, plan du texte, formulation, inscription sur le pad, révision et processus d'écriture.

Comme l'explique à juste titre Dejean (2003) qui compare des rédactions à deux sur papier et sous Word ${ }^{\circ}$, l'écran a l'avantage de rendre le texte en permanence accessible aux co-scripteurs. Nous l'avons observé dans notre dispositif, mais avec des différences notables entre Word $^{\circ}$ et Framapad $^{\circ}$, notamment au niveau de l'inscription et de la révision. En effet, contrairement au dispositif avec Word ${ }^{\circ}$ où il n'y a qu'un ordinateur par groupe, Framapad ${ }^{\circ}$ permet la co-réalisation de l'inscription par tous les apprenants du groupe, étant donné que chacun a un ordinateur et peut rédiger sur le pad où et quand il le veut. De plus, l'expertise de chaque scripteur peut en principe s'exercer plus facilement via des hétéro-révisions (révisions par un co-apprenant) faites directement sur le pad. Nous allons donc nous arrêter sur chacune de ces spécificités de Framapad ${ }^{\circ}$. 


\subsection{La co-réalisation de l'inscription avec Framapad ${ }^{\circledR}$}

Avec Framapad ${ }^{\circledR}$, les apprenants tirent parti de la fonctionnalité collaborative qui permet à chacun de rédiger quand il le veut ${ }^{15}$, alors qu'avec Word ${ }^{\circ}$ le scripteur tend à rester le même tout au long de la rédaction du texte.

En effet, sur les 15 textes rédigés en collaboration avec Framapad ${ }^{\circledR}$, on note que presque tous (sauf le texte $1 \mathrm{du}$ groupe 3 ) ont été co-inscrits par plusieurs étudiants. Le plus souvent, tous les étudiants du groupe participent à l'inscription du texte, même si l'un d'eux inscrit en général plus que les autres ${ }^{16}$; ce scripteur principal est d'ailleurs stable d'un texte à l'autre.

21 Cette co-inscription sur le pad se fait selon différentes modalités :

- soit de manière successive (5 textes sur 14) : les étudiants inscrivent à tour de rôle sur le pad;

- soit de manière simultanée ( 2 textes sur 14$)$ : les étudiants inscrivent en même temps à des endroits différents du pad, chacun prenant donc en charge certains éléments du texte (syntagme, phrase, paragraphe);

- soit de manière « mixte », tantôt successive, tantôt simultanée (7 textes sur 14).

Dans notre corpus, c'est la modalité d'inscription mixte qui est la plus fréquente, celle qui inclut un recours aux deux modalités.

Avec la modalité successive, l'inscription du texte est comme décomposée : chaque segment de texte (syntagme, phrase, groupe de phrases et/ou paragraphe) est inscrit par un apprenant différent. Voici un exemple de phrase co-inscrite (l'indication du scripteur suit le fragment) : " Par rapport à [scripteur 1] l'article d'Agnès Leclair sur le café Joyeux [scripteur 2], publié au mardi 20 mars 2018 qui [scripteur 1], aborde un grand sujet [scripteur 3] [...]» (groupe 5, texte 1). Cela reflète très exactement la co-réalisation de tâches convergentes et l'expertise distribuée qui caractérisent la littératie numérique (cf. partie 1). De manière générale, cette alternance des scripteurs n'a pas d'effets négatifs sur la cohérence ou la cohésion du texte, bien au contraire (les annotations de l'enseignant ne portent pas mention de tels effets), sans doute parce que les apprenants, qui ont chacun le texte sous les yeux, ne perdent pas de vue leur objectif commun et s'efforcent donc de relier chacun de leurs apports au texte déjà rédigé.

Dans notre corpus, la modalité d'inscription simultanée, en revanche, débouche parfois sur des textes mal formés (4 textes sur 15). Cette modalité d'inscription est privilégiée notamment par les étudiants du groupe 4 qui se répartissent les parties du texte à écrire ( $2^{\mathrm{e}}$ partie du texte 1 , textes 2 et 3 ), puis les rédigent individuellement : le fait que l'enregistrement présente de longs moments de silence et l'absence d'hétéro-révisions sur le pad montrent que ces étudiants collaborent très peu pour la formulation et la révision de chaque partie rédigée. Il en résulte trois textes manquant de cohérence et de cohésion et présentant des passages peu compréhensibles. Cette stratégie de rédaction peu payante s'apparente à une "inscription en parallèle ", c'est-à-dire à une juxtaposition de textes sans travail de lissage, ni amélioration par un pair de chaque partie inscrite. Il existe cependant dans notre corpus des cas de modalité mixte (donc incluant de la modalité simultanée) qui produisent des textes bien formés : lorsque le recours à la modalité mixte est accompagné d'une relecture du texte dans son ensemble et d'hétéro-révisions (les 3 textes du groupe 2 et le texte 2 du groupe 3 ). 

collaboration. On observe que ces révisions par un pair sont souvent effectuées pendant qu'un autre étudiant inscrit le texte commun et se font donc sans verbalisation. Cette fonctionnalité permet à un étudiant qui intervient peu d'augmenter sa participation, comme Joyce du groupe 3 lors de la rédaction du texte 1 : alors que Samir inscrit seul le texte en échangeant par oral surtout avec Zara, l'historique dynamique révèle que Joyce fait plusieurs corrections locales en silence. La coprésence des scripteurs semble donc faciliter la co-écriture dans la mesure où elle ne provoque pas de réticences à intervenir sur un segment de texte inscrit par un autre, contrairement à ce que l'on constate souvent lors d'une co-élaboration de texte à distance (Elola \& Oskoz, 2010 ; Ware \& O'Dowd, 2008). Ollivier (2016) considère ce type de réticences comme le signe d'enjeux de pouvoir qui se manifestent dans les prises de parole.

En résumé, la plupart des groupes d'apprenants (sauf le groupe 4) de notre dispositif exploitent les fonctionnalités collaboratives de Framapad ${ }^{\circ}$ et coréalisent ainsi leur texte aux niveaux de l'inscription et de la révision; les hétéro-révisions qu'ils font à l'écrit comme à l'oral améliorent les textes et révèlent l'expertise de chacun. De plus, on a vu que les spécificités du logiciel collaboratif permettent à certains étudiants, qui 
interviennent moins que leurs pairs lors de la rédaction à plusieurs, d'augmenter leur collaboration.

31 On constate ici une grande différence entre le processus d'écriture sous Word ${ }^{\circ}$ et celui avec Framapad. Dans le premier cas, la technicité du logiciel facilite les opérations de reformulation, suppression, déplacement et la fonctionnalité " révision » avec le possible ajout de commentaires ${ }^{19}$ permet à chacun de contribuer à l'écriture du texte. Mais il s'agit alors d'un mode d'intervention différé, qui apparait en marge du texte et qui, de plus, peut être ou non accepté par les co-scripteurs. Framapad ${ }^{\circ}$, au contraire, permet aux co-scripteurs d'intervenir directement dans le texte et de façon concomitante : l'inscription d'une "retouche » n'est pas médiée par la seule personne manipulant le logiciel; chaque scripteur est responsable de son inscription et se trouve ainsi responsabilisé. De plus, sa manipulation devient en quelque sorte opérationnelle : elle oblige les co-scripteurs à veiller aux modifications qui s'opèrent dans la temporalité même de leur déroulement. La participation de chacun n'est pas séquentielle mais simultanée. On peut donc dire que Framapad ${ }^{\circ}$ met en scène l'expertise distribuée.

Nous avons vu que certaines participations entrainent une verbalisation. Il y a en effet de nombreuses négociations entre co-apprenants.

\section{Les négociations lors de l'écriture collaborative en présentiel}

Pour qu'il y ait négociation, il faut qu'il y ait conflit et coopération. Dans notre dispositif, les échanges entre les apprenants concernent de nombreux domaines: le respect de la consigne et du genre textuel attendu, l'organisation du travail de groupe ainsi que toutes les composantes de l'élaboration textuelle (cf. Hidden \& Portine, 2020), mais les désaccords à proprement parler se situent avant tout aux niveaux de la recherche d'idées et de la formulation. C'est pourquoi nous allons focaliser notre attention sur ces deux aspects du processus rédactionnel. L'analyse du corpus permet d'observer comment les apprenants opèrent les négociations afin de parvenir à la rédaction d'un texte commun, ce qui est toujours le cas, sauf pour le texte $1 \mathrm{du}$ groupe $5^{20}$.

\subsection{Les négociations au niveau de la recherche d'idées}

Dans tous les groupes, la phase proprement dite de rédaction est le plus souvent, mais pas exclusivement, précédée par une phase de négociations au cours de laquelle les étudiants s'interrogent sur leurs opinions respectives (élaboration d'une opinion commune au sein de la micro-communauté en cours de construction) et parfois se mettent aussi d'accord sur un plan de texte. Ces échanges sont nécessaires, car les étudiants ne partagent pas toujours le même avis sur la question à traiter. Cette phase de concertation est plus ou moins longue, allant de quelques minutes au tiers du temps de rédaction (rédaction du texte 2 du groupe 2), ce qui est assez conséquent.

Par exemple, lors de la rédaction du texte 1 par le groupe 1, les étudiants listent leurs arguments sur le pad avant de commencer à rédiger ${ }^{21}$ : ils énumèrent des avantages, puis lorsqu’Emma propose de considérer aussi les désavantages (" parce que on pense 
que c'est une bonne idée, mais peut-être il y a aussi des désavantages »), elle doit convaincre ses pairs qui n'en voient pas, ce qui donne lieu à une discussion pendant environ 4 minutes; l'ensemble des négociations auxquelles participent les trois étudiants dure environ 10 minutes, soit le quart du temps de rédaction. Certains groupes éprouvent d'ailleurs le besoin de résumer l'opinion commune qu'ils ont réussi à élaborer avant de commencer à rédiger (recherche de convergence).

\subsection{Les négociations au niveau de la formulation} échanges sur la formulation occupent souvent une grande place dans la conversation rédactionnelle, sans doute parce que les étudiants doivent écrire dans une langue qu'ils ne maitrisent pas encore très bien : l'écriture est souvent interrompue par des doutes sur la formulation ("c'est quoi la phrase? » pour demander comment continuer une phrase) ainsi que par des questions sur la langue qui proviennent soit du scripteur, soit d'un pair et qui portent sur le choix d'un déterminant, sur la place d'un mot dans la phrase, sur un choix lexical, sur l'orthographe, la ponctuation, etc. Ces échanges peuvent durer plusieurs minutes lorsque les étudiants ne parviennent pas à trouver la solution et finissent par vérifier dans le dictionnaire, sur un site web ou en faisant appel à l'enseignant. Par exemple, lors de la rédaction du texte 1 par le groupe 3 , Zara dicte à Samir le segment de phrase "Ça leur permet à devenir»; celui-ci corrige la préposition en disant "permet d'être ", puis un commentaire métalinguistique: "permettre de, ce verbe prend le de ", mais il faut plusieurs tours de parole avant que Zara accepte cette révision (elle répète plusieurs fois «à devenir " avant de dire finalement « de devenir »).

Lors de la rédaction du texte 2 par le groupe 2, Kader en relisant la phrase écrite sur le pad «On voudrait dire qu'on est d'accord avec votre avis», propose de remplacer « qu'on » par « que l'on ». Ses deux co-apprenantes refusent, Cho répondant «non, c'est pas correct ». Kader insiste puis appelle l'enseignant pour les départager; ce dernier leur expliquant que c'est possible, mais un peu recherché, il s'ensuit de nombreux échanges entre les étudiants pour savoir si la formulation proposée par Kader est appropriée ou non, le tout durant pas moins de 2 minutes.

Ces échanges sont précieux, car ils montrent encore une fois comment l'expertise de chaque co-apprenant au sein des groupes contribue à l'élaboration du texte commun.

\section{Conclusion}

Dans les limites de cet article, nous avons cherché à mettre en lumière les apports d'un outil numérique à l'apprentissage de la rédaction en langue étrangère en montrant comment il peut faciliter une réelle collaboration entre apprenants (co-réalisation de tâches convergentes, co-engagement dans le processus rédactionnel et expertise distribuée) lors de l'écriture d'un texte à plusieurs, conformément à une approche de type littératie: en s'engageant pleinement dans la tâche d'écriture (formulation et inscription) et de réécriture (révisions), la plupart des co-apprenants montrent qu'ils considèrent le texte commun comme leur et assument donc pleinement leur posture de sujet-scripteur. 
La plupart de nos apprenants se saisissent des fonctionnalités collaboratives du logiciel, mais on peut se demander si ce n'est pas dû au fait que notre dispositif était en présentiel. En effet, une étude exploratoire ${ }^{22}$ sur l'utilisation de Framapad ${ }^{\circ}$ à distance montre que plusieurs groupes d'apprenants ne s'approprient pas le logiciel (ils écrivent sur un autre support puis copient et collent leur texte sur le pad) et interviennent très peu, voire pas du tout sur les parties de texte rédigées par leurs pairs. On retrouve ici la question de réticences éventuelles (cf. supra) dans les modalités d'appropriation des fonctionnalités collaboratives du logiciel par les étudiants.

\section{BIBLIOGRAPHIE}

BAWDEN, David. (2001). Information and Digital Literacies: A Review of Concepts. Journal of Documentation, 57(2), 218-259.

BAWDEN, David. (2008). Origins and Concepts of Digital Literacy. Dans C. Lankshear \& M. Knobel (dir.), Digital Literacies: Concepts, Policies and Practices (p. 17-32). Peter Lang.

BAWDEN, David \& RoBInSON, Lyn. (2002). Promoting Literacy in a Digital Age. Learned Publishing, 15(4), 297-301.

CAMBRIDGE AsSESSMENT. (2013). What Is literacy? An Investigation into Definitions of English as a Subject and the Relationship between English, Literacy and 'Being Literate'.

$<w w w . c a m b r i d g e a s s e s s m e n t . o r g . u k / I m a g e s / 130433-w h a t-i s-l i t e r a c y-a n-i n v e s t i g a t i o n-i n t o-$ definitions-of-english-as-a-subject-and-the-relationship-between-english-literacy-and-beingliterate-.pdf>.

DEJEAN, Charlotte. (2003). Rédactions conversationnelles sur papier et sur ordinateur : une étude de cas. Alsic, 6(1). <https://doi.org/10.4000/alsic.2179>.

Depover, Christian, De Lièvre, Bruno, Peraya, Daniel, Quintin, Jean-Jacques \& JaILlet, Alain (dir.). (2011). Le tutorat en format à distance. De Boeck.

ElolA, Idoia \& OsKoz, Ana. (2010). Collaborative Writing: Fostering Foreign Language and Writing Conventions Development. Language Learning \& Technology, 14(3), 51-71.<www.lltjournal.org/ item/2700>.

FAYOL, Michel. (2013). L'acquisition de l'écrit. Presses universitaires de France.

GAUlmyn, Marie-Madeleine DE, Bouchard, Robert \& RABATEL, Alain. (2001). Introduction. Dans M.M. de Gaulmyn, R. Bouchard \& A. Rabatel (dir.), Le processus rédactionnel. Écrire à plusieurs voix (p. 9-16). L'Harmattan.

GAULMYN, Marie-Madeleine DE. (2005). Le corpus comme un tout. Dans R. Bouchard \& L. Mondada (dir.), Les processus de la rédaction collaborative (p. 17-54). L'Harmattan.

GILSTER, Paul. (1997). Digital literacy. Wiley.

GILSTER, Paul. (2006). Digital Fusion: Defining the Intersection of Content and Communications. Dans A. Martin \& D. Madigan (dir.), Digital Literacies for Learning (p. 42-50). Facet Publishing. 
Goody, Jack \& WATT, Ian. (1963). The Consequences of Literacy. Comparative Studies in Society and History, 5(3), 304-345.

GrosBoIs, Muriel. (2012). Didactique des langues et technologies : de l'EAO aux réseaux sociaux. Presses de l'Université Paris-Sorbonne.

HIDDEN, Marie-Odile. (2018). Apprendre à réviser son texte en langue étrangère : les effets d'une révision guidée. Dans J. Demperio, M. Deraiche, R. Dewart \& B. Zuercher (dir.), L'enseignementapprentissage de l'écrit : état des lieux (p. 33-46). Université du Québec à Montréal.

HIDDEN, Marie-Odile \& PORTINE, Henri. (2017). L'apprenant en FLE confronté à la polyphonie textuelle : littéracie, discours, grammaire. Lidil, 56. <https://doi.org/10.4000/lidil.4766>.

HIDDEN, Marie-Odile \& PoRTINE, Henri. (2020). Des pratiques collaboratives rédactionnelles en (français) langue étrangère à l'appropriation individuelle de l'écrit. Travaux de didactique du français langue étrangère, 76. <https://revue-tdfle.fr/articles/revue-76/1541-des-pratiquescollaboratives-redactionnelles-en-francais-langue-etrangere-a-l-appropriation-individuelle-de-1ecrit>.

KERN, Richard. (2000). Literacy and Language Teaching. Oxford University Press.

KuHn, Thomas S. (1970). The Structure of Scientific Revolutions (2e éd.). The University of Chicago Press.

LANHAM, Richard. (1995). Digital Literacy. Scientific American, 273(3), 198-200.

LANKSHEAR, Colin \& KNOBEL, Michele. (2003). New Literacies: Changing Knowledge and Classroom Learning. Open University Press.

LANKSHEAR, Colin \& KNOBEL, Michele. (2006). New Literacies: Everyday Practices and Classroom Learning ( $2^{e}$ éd.). McGraw-Hill \& Open University Press.

MANGENOT, François. (2000). Contexte et conditions pour une réelle production d'écrits en ALAO. Alsic, 3(2), 187-206. <https://doi.org/10.4000/alsic.1833>.

MARTIN, Allan \& GRUDZIECKI, Jan. (2006). DigEuLit: Concepts and Tools for Digital Literacy Development. Innovation in Teaching and Learning in Information and Computer Sciences, 5(4), 249-267. <https://doi.org/10.11120/ital.2006.05040249>.

MARTIN, Allan \& MADIGAN, Dan. (2006). Digital Literacies for Learning. Facet Publishing.

MASSERon, Caroline \& Privat, Jean-Marie (dir.). (2019). Oralité, littératie. Pratiques, 183-184. $<$ https://doi.org/10.4000/pratiques.6717>.

OCDE. (2000). La littératie à l'ère de l'information (Rapport). <www.oecd.org/fr/education/ innovation-education/39438013.pdf>.

OlLIVIER, Christian. (2016). Gestion du pouvoir dans la co-construction de savoirs. Distances et médiations des savoirs, 15. <https://doi.org/10.4000/dms.1558>.

OLLIVIER, Christian. (2018). Littératie numérique et approche socio-interactionnelle pour l'enseignementapprentissage des langues. Centre européen pour les langues vivantes. <www.ecml.at/Portals/1/ documents/ECML-resources/elang-FR-A4_28112018_112636.pdf?>.

PRIVAT, Jean-Marie \& KARA, Mohamed (dir.). (2006). La littératie autour de Jack Goody. Pratiques, 131-132. 
THORNE, Steven L. (2008). Mediating Technologies and Second Language Learning. Dans J. Coiro, M. Knobel, C. Lankshear \& D. J. Leu (dir.), Handbook of Research on New Literacies (p. 417-449). Erlbaum.

WARE, Paige D. \& O'DowD, Robert. (2008). Peer Feedback on Language Form in Telecollaboration. Language Learning and Technology, 12(1), 43-63. <www.lltjournal.org/item/2616> .

WARSCHAUER, Mark. (1999). Electronic Literacies: Language, Culture, and Power in Online Education. Lawrence Erlbaum Associates.

\section{NOTES}

1. Merci à David Bawden, Violaine Bigot, Christian Ollivier et Jean-Marie Privat pour leur apport conceptuel et à Caroline Casseville et à François Jougleux pour leur aide documentaire. Merci aux relecteurs dont les commentaires nous ont fait réfléchir à notre problématique.

2. Sur les rapports entre littératie (qui implique textualisation) et grammaire (Hidden \& Portine, 2017) et sur l'engagement actif (c'est-à-dire l'attitude proactive) que nécessite l'adaptation à une culture de l'écrit autre et la possibilité de se l'approprier pour l'apprenant(e) (Hidden \& Portine, 2020).

3. Nous nous référons à la version originale, mais signalons une version française de l'ouvrage : Thomas S. Kuhn (1972), La structure des révolutions scientifiques (L. Meyer, trad.), Flammarion. Dans la dernière édition en français (1983), la traduction a été révisée par Claude Savary.

4. National Literacy Act (États-Unis, 1991), National Literacy Strategy (Royaume-Uni, 1997, cf. le Cambridge Assessment Report, 2013). La France a manqué le coche à cette occasion, s'étant même retirée d'une enquête de l'OCDE (2000) pour se concentrer sur « les savoirs de base ».

5. Nous nous référons à l'original en anglais, mais signalons l'existence d'une traduction en français dans Privat \& Kara (2006), aux pages 31-68.

6. Même si, bien souvent, le passage à ces nouvelles façons de penser (mindset chez Lankshear \& Knobel, 2006) rendait difficile l'entrecroisement avec la problématique éducative.

7. Du point de vue de la « communauté de référence », le travail d'écriture collaboratif présente une particularité : l'écrit produit s'inscrit dans la communauté du groupe-classe (et/ou, selon les modalités de travail aussi du groupe de travail), mais vise la compréhension et l'intérêt de la communauté de ceux qui seraient les destinataires du texte « dans le réel ». Assez curieusement, on retrouve ici une problématique qui était déjà celle de la correspondance scolaire chez Freinet.

8. À distance ou non. Dans une classe, l'enseignant endosse parfois le rôle de tuteur.

9. Même s'il arrive parfois qu'une séquence orale précède une séquence écrite, comme on le verra plus loin.

10. Nous utilisons médiation et non pas médiatisation par Framapad ${ }^{\circ}$ parce qu'il est le point par lequel passent toutes les productions.

11. Voici un exemple de consigne de rédaction, suite à la lecture d'un article de la revue Science et Vie intitulé «Doit-on continuer à noter les élèves?» (25 juin 2019) : «Écrivez une lettre au courrier des lecteurs de la revue pour donner votre opinion sur cette question, en espérant que votre lettre sera publiée.»

12. Les étudiants ont aussi rédigé des textes individuels tout au long du semestre qui ont été également recueillis ainsi que les brouillons. Ces textes ne figurent pas dans le corpus ci-dessous, car leur analyse n'est pas traitée dans cet article.

13. Mangenot (2000) pointe, dans un cadre différent (la génération de textes) l'importance d'avoir, pour l'aide à l'écriture, une «bibliothèque » de textes où aller puiser des idées ou des formes de référence. Cela est proche du principe « lire pour écrire / écrire pour lire ». 
14. La seule contrainte était que les trois étudiants d'un groupe ne devaient pas avoir la même langue maternelle.

15. Néanmoins, nous allons voir que même avec Framapad ${ }^{\circ}$ il est fréquent qu'un seul apprenant écrive à partir des suggestions des autres (sorte de dictée) ou de ses propres suggestions (autodictée) : dans ce cas, une formulation à l'oral précède l'inscription proprement dite sur le pad.

16. Pour trois textes du corpus cependant, les étudiants inscrivent autant sur le pad.

17. Les prénoms des étudiants ont été changés.

18. Trois étudiants (groupe 4) ne font pas d'hétéro-révisions et une étudiante du groupe 3 fait beaucoup d'hétéro-révisions mais à l'oral.

19. Cette fonctionnalité est surtout utilisée quand l'écriture à plusieurs se fait à distance. Dans notre dispositif en présentiel, les apprenants n'y ont pas eu recours, car ils ne disposaient que d'un ordinateur par groupe: les suggestions de modifications étaient donc faites à l'oral au scripteur qui rédigeait ; là encore, il s'agissait donc d'un mode d'intervention séquentiel.

20. Le texte $1 \mathrm{du}$ groupe 5 rendu à l'enseignant diffère en effet de la dernière version du pad: cette version a été amputée d'une grande partie du dernier paragraphe, rédigé par une des étudiantes qui était souvent en désaccord avec ses co-apprenants.

21. Cette liste fonctionne comme un brouillon, car elle est effacée du pad à la fin de la rédaction.

22. Le corpus recueilli étant encore trop limité, cette recherche fera l'objet d'une publication postérieure.

\section{RÉSUMÉS}

Les auteurs présentent une recherche empirique sur une écriture collaborative en présentiel à l'aide d'un outil numérique, recherche s'inscrivant ainsi dans les réflexions théoriques sur la littératie numérique, ce qui a conduit à prendre compte les travaux anglophones sur la digital literacy. Le dispositif et le corpus sont présentés et donnent lieu à des analyses sur l'inscription, c'est-à-dire la réalisation de la trace écrite (quelle que soit la technologie), sur les révisions et sur les modalités de négociation à l'œuvre.

The authors present empirical research into face-to-face peer-collaborative writing using a digital tool, a research in relation to theoretical thoughts on digital literacy, which requires to take into account existing English-language studies into digital literacy. The device and the corpus are presented and provide analysis of the transcription, i.e. the production of the written evidence (whatever the technology), on revisions and on the modalities of negotiation for achieving the task.

\section{INDEX}

Mots-clés : littératie numérique, négociation discursive, rédaction collaborative, reformulation et révision, expertise distribuée

Keywords : digital literacy, negotiation in discourse, collaborative writing, reformulation and revision, distributed expertise 


\section{AUTEURS}

\section{MARIE-ODILE HIDDEN}

Université Bordeaux Montaigne, EA 4195 TELEM

HENRI PORTINE

Université Bordeaux Montaigne, EA 4195 TELEM 